\title{
Health Authorities Data Collection of THC:CBD Oromucosal Spray (L'Agenzia Italiana del Farmaco Web Registry): Figures after 1.5 Years
}

\author{
Francesco Patti \\ Department G.F. Ingrassia, Section of Neurosciences, University of Catania, Catania, Italy
}

\section{Key Words}

AIFA registry - Effectiveness - Multiple sclerosis spasticity ·

THC:CBD oromucosal spray $\cdot$ Tolerability

\begin{abstract}
Background: In Italy, all prescriptions for THC:CBD oromucosal spray for treatment of multiple sclerosis (MS) spasticity are linked to the official Agenzia Italiana del Farmaco (AIFA) webbased registry, which tracks the effectiveness and tolerability of medications in a prospective and observational manner. Methods: AIFA e-registry data for THC:CBD oromucosal spray collected between January 2014 and February 2015 for 1,534 patients from 30 large Italian specialized MS centres were compiled. Patients had a long disease history (17.6 \pm 8.6 years) and significant impairment (mean Expanded Disability Status Scale score $6.4 \pm 1.2$ ). MS spasticity was evaluated using the $0-10$ numerical rating scale (NRS). Results: After the first month titration and trial period, $61.9 \%$ of patients achieved sufficient improvement in spasticity ( $\geq 20 \%$ NRS) to qualify for continued treatment. After 6 months, clinically meaningful $\geq 30 \%$ NRS improvement was recorded in $40.2 \%$ of patients continuing with treatment. Spasticity-associated symptoms such as cramps and nocturnal spasms improved in most responding patients. Mean reported doses of THC:CBD oromucosal spray (6.2-6.7 sprays/day) were lower than those reported in clinical trials. Adverse events (mainly mild to moderate) were reported by $15 \%$ of patients; no new safety concerns beyond the approved label were identified. Conclusion: The results of the AIFA e-
\end{abstract}

registry analysis align with those of other THC:CBD observational projects and reaffirm the characteristics of this therapeutic option in the management of treatment-resistant MS spasticity, a frequently overlooked symptom.

c) 2016 S. Karger AG, Basel

\section{Introduction}

Over the past few years, evidence has steadily been accumulating to show that clinical outcomes with Sativex (THC:CBD) oromucosal spray in clinical practice [1-4] align closely with outcomes generated in clinical trials [5]. In view of the growing interest in observational studies, post-approval surveillance studies and patient registries, we undertook an independent project to collect clinical information on the patient population in Italy who had been prescribed THC:CBD oromucosal spray [6]. Analyses were conducted using the Agenzia Italiana del Farmaco (AIFA) government web-based registry (e-registry), which is a mandatory reporting system for all patients with treatment-resistant multiple sclerosis (MS) spasticity who are to be treated with this medication.

\section{Methods}

Since the third quarter of 2013, when THC:CBD oromucosal spray was introduced in Italy for treatment of resistant MS spasticity, data from all patients prescribed this medication

\section{KARGER}

E-Mail karger@karger.com

www.karger.com/ene
C 2016 S. Karger AG, Basel

$0014-3022 / 16 / 0757-0009 \$ 39.50 / 0$
Professor Francesco Patti

G.F. Ingrassia Department, Neuroscience Section

First Neurology Clinic Multiple Sclerosis Centre Sicilia Region, University Hospital

Catania, Via Santa Sofia 78, IT-95123 Catania (Italy)

E-Mail patti@unict.it 
Table 1. Demographic and disease characteristics of patients in the AIFA e-registry

\begin{tabular}{lc}
\hline Number of patients & 1,534 \\
Mean age, years (SD) & $51.0(9.6)$ \\
Gender, female, \% & 52.8 \\
MS type, \% & \\
$\quad$ SP & 63.7 \\
RR & 19.9 \\
PP & 16.1 \\
Mean MS duration, years (SD) & $17.6(8.6)$ \\
EDSS score, mean (SD) & $6.4(1.2)$ \\
\hline
\end{tabular}

$\mathrm{SP}=$ Secondary progressive; $\mathrm{RR}=$ relapsing remitting; $\mathrm{PP}=$ primary progressive; EDSS $=$ Expanded Disability Status Scale.

have been collected through an official Health Authorities (AIFA-ruled) e-registry. In order for physicians to gain authorization to prescribe THC:CBD oromucosal spray, certain patient selection criteria must be met. Only patients who are resistant to other medications for MS spasticity and present a score of $\geq 4$ on the spasticity $0-10$ numerical rating scale (NRS) can be prescribed THC:CBD oromucosal spray in Italy. As per the approved label, patients with severe cardiovascular disease or psychiatric disorders, pregnant women and individuals known to use psychoactive substances (e.g. street cannabis) are excluded from prescription. For eligible patients, the online data entry system also captures information regarding previous and ongoing anti-spasticity medications and other concomitant medications.

Aggregate data compiled through the AIFA e-registry are not freely accessible; access rights are restricted to a given MS centre's own data. For purposes of the current study, and subsequent to Ethics Committee approval and protection of identifiable data, an independent coordination was undertaken to compile aggregate e-registry data from 30 large MS centres across Italy (Appendix). The analysis period was January 2014 to February 2015. This first report presents results after 1 and 6 months of treatment with THC:CBD oromucosal spray. The main parameters analysed were spasticity 0-10 NRS scores at baseline, month 1 (trial period) and month 6; treatment discontinuation rates; tolerability; and daily dose of THC:CBD oromucosal spray.

\section{Results}

The demographic and disease characteristics of patients in the AIFA e-registry are summarized in table 1. In total, data for 1,534 patients from $30 \mathrm{MS}$ centres were analysed. Slightly more than half the population was female, and the mean MS duration of the sample was $17.6 \pm 8.6$ years. Approximately $80 \%$ of the population had either secondary or primary progressive disease. The mean Expanded Disability Status Scale score at baseline indicated significant disability. In the vast majority of pa-
Table 2. Reported improvement in spasticity-associated symptoms from baseline to month 1

\begin{tabular}{ll}
\hline Symptom & Improvement $^{\mathrm{a}}, \%$ \\
\hline Cramps and nocturnal spasms & 30.0 \\
Urinary dysfunction & 13.5 \\
Pain & 9.4 \\
Sleep disorder & 7.4 \\
Clonic movements & 5.5 \\
Mood disturbances & 4.6 \\
\hline
\end{tabular}

${ }^{a}$ Percent of overall sample regardless of baseline presence; multiple answers possible.

tients (94.3\%), THC:CBD oromucosal spray was added to baclofen, either alone or in combination with other antispasticity medications.

\section{Effectiveness}

A total of 1,350 of 1,534 patients reached the 1-month control visit; data for the remaining 184 patients (8.7\%) were pending at the time of analysis. In all, $61.9 \%$ of the population met the criteria for continued prescription of THC:CBD oromucosal spray ( $\geq 20 \%$ NRS improvement) and remained on treatment. About $25 \%$ of the cohort had achieved clinically relevant $\geq 30 \%$ NRS improvement at 1 month. The mean dose of THC:CBD oromucosal spray was $6.8 \pm 2.6$ sprays/day. A total of 451 patients $(29.7 \%)$ had discontinued treatment within the first 3 months of treatment, mainly due to lack of effectiveness (15\% of sample), lack of tolerability $(9.3 \%)$ or both $(2.4 \%)$.

In addition to the e-registry data, patients' medical charts were reviewed to track the evolution of symptoms associated with MS spasticity. At month 1, the presence of MS spasticity-associated symptoms was common but variable, and there was a tendency toward improvement in parallel with the reduction in spasticity (table 2).

At month 6, 599 of 1,447 possible patients were continuing with treatment, representing $39 \%$ of the original cohort. At this time point, $55.3 \%$ of patients had either discontinued treatment or data were missing; for $5.7 \%$ of patients, the time of analysis was too soon. Among the group continuing treatment with THC:CBD oromucosal spray, 225 patients ( $40.2 \%)$ showed a $\geq 30 \% \mathrm{im}$ provement in their spasticity NRS score. The mean dose of THC:CBD oromucosal spray was $6.2 \pm 2.8$ sprays/ day.
10

Eur Neurol 2016;75(suppl 1):9-12 DOI: $10.1159 / 000444236$ 
Table 3. Tolerability to THC:CBD oromucosal spray in the AIFA e-registry. Main adverse events leading to discontinuation after 1 month of treatment $(n=242)$

\begin{tabular}{ll}
\hline Adverse event & $\mathrm{n}(\%)$ \\
\hline Cognitive/psychiatric & $43(2.8)$ \\
Fatigue & $42(2.7)$ \\
Drowsiness & $41(2.7)$ \\
Dizziness & $31(2.0)$ \\
Gastrointestinal symptoms & $22(1.4)$ \\
Oral discomfort & $10(0.7)$ \\
\hline
\end{tabular}

Table 4. Comparison of baseline features of the patient populations and key findings

\begin{tabular}{lccc}
\hline Parameter & $\begin{array}{c}\text { MOVE 2 } \\
\text { Germany [3] }\end{array}$ & RCT [5] & AIFA e-registry [6] \\
\hline Number of patients, $\mathrm{n}$ & 300 & 572 & 1,534 \\
Female, n (\%) & $168(60.9)$ & $347(61)$ & $810(52.8)$ \\
Age, years (SD) & $50.0(9.4)$ & $48.9(9.6)$ & $51(9.6)$ \\
Mean MS duration, years (SD) & $15.4(9.0)$ & $12.4(7.7)$ & $17.6(8.6)$ \\
Baseline EDSS score, mean (SD) & $6.0(1-9)^{\dagger}$ & $6.0(1.4)$ & $6.4(1.2)$ \\
NRS T0 in initial responders, mean (SD) & $6.4(1.8)$ & $6.9(1.2)$ & $7.6(1.4)$ \\
NRS T1 in initial responders, mean (SD) & $3.9(1.5)$ & $3.9(1.5)$ & $5.3(1.3)$ \\
Percentage reduction T0-T1 in initial responders, \% & 40.2 & 43.5 & 30.3 \\
$\geq 20 \%$ NRS response, month 1, \% & 42 & 47 & 70.3 \\
$\geq 30 \%$ NRS response, month 3, \% & 41 & 36 & $27.9(40.2 \%$ at month 6) \\
Mean dose, sprays/day & 6.7 & 8.3 & 6.8 \\
AEs (1 or more), \% & 16.6 & 46.9 & 15.9
\end{tabular}

EDSS $=$ Expanded Disability Status Scale

${ }^{\dagger}$ Median score.

\section{Tolerability}

The main types of adverse events (AEs) reported by patients are summarized in table 3. No safety concerns beyond the approved label for THC:CBD oromucosal spray were identified and there was no evidence of abuse/ misuse.

Five serious AEs were reported across the study $(0.3 \%$ of overall sample), which included 1 report each of renal failure, death due to acute myocardial infarction, hypertensive crisis, laryngeal carcinoma and breast cancer. None of these events was considered related to THC:CBD oromucosal spray.

\section{Inter-Study Comparison}

To determine the relationship between data collected in the AIFA e-registry and those from other available studies of THC:CBD oromucosal spray, we compared our findings with the published observational Mobility Improvement 2 (MOVE) 2 study from Germany [3] and the enriched-design randomized controlled trial (RCT) of Novotna et al.
[5] (table 4). Relative to the MOVE 2-Germany and RCT populations, AIFA e-registry patients had a longer disease duration, greater degree of disability and more severe MS spasticity. Nevertheless, a higher proportion of patients in the AIFA e-registry achieved an initial response to THC:CBD oromucosal spray at month 1 (70.3 vs. 42 vs. $47 \%)$, mirroring the results of the recently published interim analysis of MOVE 2-Italy in which an initial response in $82.9 \%$ of the population was documented [4]. Also similar to MOVE 2-Italy, the 30\% responder rate at month 3 was lower in the AIFA population than in MOVE 2-Germany and the RCT (27.9 vs. 41 vs. $36 \%$ ), but increased to $40.2 \%$ among patients $(n=559)$ who had reached the month 6 visit at the time of analysis, suggesting that additional time may be required to achieve a clinically meaningful outcome in the type of patient included in the AIFA e-registry sample. Notably, observational studies including the AIFA e-registry analysis suggest that a relevant proportion of patients treated in everyday practice gain meaningful symptomatic relief of spasticity with THC:CBD 
oromucosal spray at a dose of around 6 sprays/day, possibly even lower with continued use. Moreover, THC:CBD oromucosal spray is well tolerated during everyday use.

\section{Conclusions}

The AIFA e-registry population ( $\mathrm{n}=1,534$ patients) is the largest single sample of patients prescribed THC:CBD oromucosal spray analysed to date. Consistent with results of other patient registries, observational studies and a pivotal phase III RCT of THC:CBD oromucosal spray, the medication showed good effectiveness and tolerability in the management of patients with resistant MS spasticity. Strict adherence to the trial of therapy approach can assist in the selection of patients who respond to treatment, thus limiting exposure to patients most likely to benefit and reducing the economic burden of THC:CBD oromucosal spray on healthcare systems. Importantly, patients' degree of improvement in spasticity was similar to or slightly better in daily practice than that demonstrated in clinical trials and was achieved at lower mean doses. Across the full range of studies of THC:CBD oromucosal spray, including those reporting on wider use in everyday practice, no evidence of risks related to herbal cannabis such as abuse/diversion has been identified.

\section{Disclosure Statement}

F. Patti reports for the past year speaking and advisory board activities for Almirall, Bayer, Biogen, Merck Serono, Novartis, Sanofi-Genzyme and Teva; research grants from FISM, MIUR and University of Catania.

\section{Appendix}

The following collaborating centers are gratefully acknowledged.

\begin{tabular}{|c|c|}
\hline Investigator & Location \\
\hline Maria Pia Amato & Firenze \\
\hline Maria Donata Benedetti & Verona \\
\hline Roberto Bergamaschi & Pavia \\
\hline Antonio Bertolotto & Orbassano \\
\hline Simona Bonavita & Napoli \\
\hline Vincenzo Brescia Morra & Napoli \\
\hline Roberto Bruno Bossio & Cosenza \\
\hline Paola Cavalla & Torino \\
\hline Diego Centonze & Roma \\
\hline Giancarlo Comi & Milano \\
\hline Salvatore Cottone & Palermo \\
\hline Maria Chiara Danni & Ancona \\
\hline Ada Francia & Roma \\
\hline Aurora Fuiani & Foggia \\
\hline Claudio Gasperini & Roma \\
\hline Angelo Ghezzi & Gallarate \\
\hline Alfonso Iudice & Pisa \\
\hline Giacomo Lus & Napoli \\
\hline Giorgia Teresa Maniscalco & Napoli \\
\hline Maria Giovanna Marrosu & Cagliari \\
\hline Silvia Messina & Catania \\
\hline Massimilliano Mirabella & Roma \\
\hline Enrico Montanari & Parma \\
\hline Francesco Patti & Catania \\
\hline Carlo Pozzilli & Roma \\
\hline Marco Rovaris & Milano \\
\hline Eduardo Sessa & Messina \\
\hline Claudio Solaro & Genova \\
\hline Daniele Spitaleri & Avellino \\
\hline Maria Trojano & Bari \\
\hline Paola Valentino & Catanzaro \\
\hline Mario Zappia & Catania \\
\hline
\end{tabular}

\section{References}

1 Etges T, Taylor A, Karolia K, Lauder H, Daka B, Wright S: A final report of an observational post-marketing safety registry of patients primarily from the United Kingdom who have been prescribed an oromucosal spray containing $\Delta 9$-tetrahydrocannabinol and cannabidiol (THC:CBD). Mult Scler 2015; 21(suppl 11):610

2 Oreja-Guevara C: Observational safety study of THC:CBD oromucosal spray (Sativex) in multiple sclerosis patients with spasticity. Clin Exp Pharmacol 2015;5:184.

3 Flachenecker P, Henze T, Zettl UK: Nabiximols (THC/CBD oromucosal spray, Sativex ${ }^{\circledR}$ ) in clinical practice - results of a multicenter, non-interventional study (MOVE 2) in patients with multiple sclerosis spasticity. Eur Neurol 2014;71:271-279.

4 Trojano M, Vila C: Effectiveness and tolerability of THC/CBD oromucosal spray for multiple sclerosis spasticity in Italy: first data from a large observational study. Eur Neurol 2015;74:178-185.

5 Novotna A, Mares J, Ratcliffe S, Novakova I, Vachova M, Zapletalova O, Gasperini C, Pozzilli C, Cefaro L, Comi G, Rossi P, Ambler Z, Stelmasiak Z, Erdmann A, Montalban X, Klimek A, Davies P; Sativex Spasticity Study
Group: A randomized, double-blind, placebo-controlled, parallel-group, enriched-design study of nabiximols* (Sativex $\left({ }^{\circledR}\right)$ ), as add-on therapy, in subjects with refractory spasticity caused by multiple sclerosis. Eur J Neurol 2011;18:1122-1131.

6 Patti F, Messina S, Amato MP, Benedetti $\mathrm{MD}$, Bergamaschi R, Bertolotto A, et al: Multicenter, Prospective, Observational Study Aimed at Evaluating Sativex Effects (Effectiveness and Tolerability) in a Large Population of Italian Multiple Sclerosis Patients: SAFE Study. Spain, Barcelona, 2015, pp 7-10. 